\title{
Every Six Months
}

National Cancer Institute

\section{Source}

National Cancer Institute. Every Six Months. NCI Thesaurus. Code C89789.

To be done at regular intervals of every six months. 